\title{
Necrosis BRONJ of the mandible, complicated by Actinomyces infection - Case report
}

\author{
Anna Gaweda', Agnieszka Kałuża², Jolanta Wojciechowicz' ${ }^{1}$, Mateusz Wachowicz², Sara Dweik ${ }^{2}$ \\ ${ }^{1}$ Department of Maxillofacial Surgery. Medical University, Lublin, Poland \\ ${ }^{2}$ Medical University, Lublin, Poland
}

Gaweda A, Kałuża A, Wojciechowicz J, Wachowicz M, Dweik S. Necrosis BRONJ of the mandible, complicated by Actinomyces infection - Case report. J Pre-Clin Clin Res. 2018; 12(3): 82-86. doi: 10.26444/jpccr/94379

\begin{abstract}
I Abstract
Biphosphonate-related osteonecrosis of the jaw (BRONJ) is a common complication of the therapy of malignant cancers. Drugs improve bone density and reduce hypercalcaemia in patients with primary tumours, as well as their metastases to the bones. A characteristic feature are emerging foci of osteonecrosis of the jaws, with secondary infection which is often actinomycotic. Actinomyces are Gramm-positive bacteria which colonize the mucous membrane and in favourable conditions are the cause of inflammation. BRONJ appears spontaneously or may be a consequence of surgical treatment in the area of the jaws. The case is presented of an 82-year-old woman after hysterectomy due to a cancerous lesion who had taken ibandronic acid for a long time. Because of the severe pain in the mandible and the advanced disease, surgical sequestration of the necrosis and antibiotic treatment of the actinomycosis were necessary.
\end{abstract}

I Key words

bisphosphonate-related osteonecrosis of the jaws, biphosphonates, Actinomycosis, surgical debridement, antibiotic therapy

\section{INTRODUCTION}

BRONJ (Biphosphonate Related Osteonecrosis of the Jaws) may appear spontaneously, but most often it is caused by local trauma, such as tooth extraction, irritation by a prosthesic plate, periodontal surgeries, implant placement, marginal or apical periodontitis. In all these cases, local inflammation occurs, caused by disruption of the mucous membrane or bone contamination. In the organism of persons who do not take biophosphates (BP's), it triggers the bone healing processes by increasing the activity of osteoclasts, which leads to its repair.

Bisphosphonates are synthetic derivatives of inorganic pyrophosphates - regulators of bone mineralization. [1] The mechanism of their action is mainly based on the inhibition of osteoclast functions, which are responsible for the processes of bone destruction, as well as its remodeling. [2] The main indications for their administration are osteoporosis, osteopenia, osteogenesis imperfecta, Paget's disease, as well as the occurrence of cancer metastases (breast, prostate, lung, multiple myeloma) to the bone, or their prevention. $[3,4]$ The incidence of BRONJ is estimated at $0.03-0.1 \%$. [5]

The main theories for the occurence of BRONJ concern the disorder of osteoclast function by bisphosphonates, decrease of angiogenesis and reduction of blood flow through the bone, direct toxicity and local disturbances in immune balance. Necrosis occurs almost exclusively s within the craniofacial bones - the mandible and maxilla [6]. This is probably due to increased remodeling in the above-mentioned bones and higher activity of osteoclasts, to which BP's have an affinity. In addition, the oral cavity is a specific environment, because the mucous membrane is a weaker barrier than the skin, moreover, it is the site in which diverse microflora exist. [7]

Address for correspondence: Anna Gaweda, Department of Maxillofacial Surgery. Medical University of Lublin, The Medical University of Lublin, Staszica 11, 20-081 Lublin, Poland, 20-081 Lublin, Poland

E-mail: agaweda5@wp.pl

Received: 1 June 2018; Accepted: 16 august 2018
The BRONJ foci are prone to infection with the Actinomyces genus, a Gramm-positive, opportunistic bacteria that grows in anaerobic conditions. This saprophytic microorganism belongs to the flora residing in the oral cavity (in carious lesions, periapical lesions, gingival pockets), as well as the gastrointestinal and respiratory tract. There is no possibility of penetration through the undamaged mucous membrane. Its continuity is interrupted during, e.g., extraction or other surgical procedures. In terms of necrosis of the facial bones, the bacteria easily penetrates into the deeper layers, causing infection and osteitis $[2,6,8]$. Actinomycosis is primarily a chronic specific inflammation. The main pathogen is Actinomyces israeli, but the presence of A. viscosus, A. naeslundi, A. odontolyticus is also observed [9]. Any damage to the mucous membrane in mouth allows the bacteria to penetrate into deeper tissues and cause inflammation $[10,11]$.

Additionally, the general condition of the patient also affects the occurrence of Actinomycosis - people with coexisting systemic diseases that disrupt the function of the immune system are more predisposed. Clinical symptoms of Actinomycosis are leatherlike infiltration of subcutaneous tissue with forming fistulas, from which characteristic yellowgreen clumps are filtered $[10,11]$ Infection can be spread to the bone, which in the case of cervical-facial Actinomycosis, is most often the mandible [9]. In this case, on radiological images an osteolytic defect can be observed. Diagnostic is not easy due to the rigorous conditions of transport, storage and Actinomycis laboratory culture; therefore, it is necessary to perform additional histopathological examination which allows visualization of the colonies of Actinomycetes. Treatment includes antibiotic therapy - mainly penicillin and surgical treatment [12]. Actinomycotic infection is associated with an uncertain prognosis after surgical treatment and the risk of BRONJ recurrence [2]. Surgery procedure without antimicrobial therapy will not be beneficial if BRONJ is complicated by Actinomyces infection [8]. The first-line drug is penicillin $\mathrm{G}$, administered intravenously at a dose 
of 10-20 million IU / day for 2-6 weeks, followed by phenoxypenicyllin orally (2-4 g/ day) for several weeks [9].

Bisphosphonates, as inhibitors of osteoclastic cells, do not allow for normal bone regeneration $[1,2,6,9,10]$. Additional risk factors are the duration of bisphosphonate therapy [11], as well as the route of administration, which is also a factor affecting the risk of osteonecrosis. The risk increases in patients taking intravenous bisphosphonates, and in the case of oral therapy, the chances of BRONJ occurring are lower $[4,9,10,11,12,13,14]$. It is thought that this is due to the fact that after intravenous administration of $50 \%$ of the drug is delivered to the bones, while only $1 \%$ of the drug is absorbed from the gastrointestinal tract and proportionally less reaches the bones $[1,13]$. Gender observation shows that BRONJ affects women more often, but it is possible that this is associated with primary disease - osteoporosis, which affects women more often, especially in postmenopausal age, as well as metastases of breast cancer $[14,15]$.

The diagnosis of BRONJ is made in the case of the appearance of exposed bone in the maxillo-facial area which does not heal for more than eight weeks. In the interview, the patient gives current or past treatment with bisphosphonates, as well as the lack of radiotherapy in this region. It is also necessary to exclude the presence of malignant metastases to the mandible or maxilla [6]. To facilitate the diagnosis and determine the degree of disease development, the following classification system for BRONJ has been devised:

- Patients at risk - taking BF's but no symptoms of exposed bone.

- Grade 0 - no exposed bones, symptoms such as odontalgia, maxillary sinus pain, tooth loss not caused by periodontisis, visible changes on the radiograph, such as bone resorption without periodontal reasons, disturbance of bone trabeculum, areas of osteosclerosis, widening of the periodontium.

- Grade 1 - exposed bone, no symptoms of inflammation, lesions can be observed in the radiograph.

- Grade 2 - exposed bone, pain symptoms, inflammation symptoms, lesions observed on the radiograph.

- Grade 3 - exposed bone, pain symptoms, symptoms of inflammation, lesions on the radiogram, as well as, among others: progression of necrosis towards the mandible, maxillary sinus, zygomatic bone, pathological fracture, extraoral fistula, oro-sinus or oronasal connection, osteolysis spreading to the lower margin of the jaw or the fundus of the maxillary sinus (6).

Clinical symptoms of BRONJ should be confirmed by radiological examination. In the first place, a panoramic radiograph is taken which, however, does not allow determination of the location of the lesion in three dimensions. Therefore, cone beam computed tomography (CBCT), computed tomography (CT) or magnetic resonance tomography (MR) are useful in planning treatment. These studies may reveal an increased or decreased density of bone, periosteal reactions or the presence of bone sequestrum. They can also confirm bone involvement in the absence of its denudation due to increased water content, which is recorded by T1-dependent images (hypointense) and T2dependent (hyperintense). Scintigraphy, PET or SPECT are also useful $[4,7,16]$.

BRONJ treatment depends on the level of advancement. Patients who lack any symptoms (at risk) must be informed about the risks and instructed to maintain proper oral hygiene. At stage 0 , systemic management is recommended - analgesics and antibiotics. In patients with bone exposed, but without other ailments (stage 1), conservative therapy is used, such as rinsing the mouth with antiseptic rinses, e.g. $0.12 \%$ chlorhexidine or $2 \%$ potassium iodide [17]. Stage 2 treatment - exposed bone, combined with infection and pain, is widely discussed. The American Association of Oral and Maxillofacial Surgeons recommends the introduction of antibiotic treatment (penicillin with clavulonic acid, metronidazole, doxycycline, clindamycin) [6], analgesics, mouthwash with antiseptic solutions, as well as debridement wounds from necrotic tissue and smoothing sharp bone edges. The most advanced stage (stage 3 ) uses antibiotic therapy, analgesics, rinsing with antimicrobial solutions, but the main method of treatment is sequestromy and resection of the affected bone [6].

Preventive treatment is very important in patients who have decided to undergo bisphosphonate therapy. The most important activity is to conduct a thorough dental examination. Finding information about the presence of teeth qualifying for extraction in the oral cavity, with gangrenous pulp, with large periapical changes, residual roots and teeth with acute periodontitis. In addition, if the patient uses a prosthesis, its adhesion should be checked as well as irritation of the mucous membrane leading to its injuries. Periodontal inflammation should be cured. If general conditions permit, BF's therapy should be deferred until oral cavity treatment is performed for 2-3 weeks, or until healing has been achieved. [6] The patient must be instructed in the matter of maintaining oral hygiene, instructed in hygiene and control of the quality of hygienic procedures performed.

\section{CASE REPORT}

The patient, an 82-year-old female, reported to the Clinic of Maxillofacial Surgery in Lublin because of inflammation in the anterior part of the mandibular bone. In the interview, she referred to pain and oedema of the alveolar bone in the region of tooth 43 and in the mentum area. The complaints intensified after tooth extraction. In the intraoral examination, an area of $3 \times 1.5 \mathrm{~cm}$ of mandibular bone was exposed near alveolus 43 , and abundant, purulent exudation from the lesion was seen. Fetor ex ore was perceptible. The surrounding gum was swollen and tender in palpation (Fig. 1).

The general medical history showed that the patient had undergone hysterectomy about 10 years ago due to cancer of

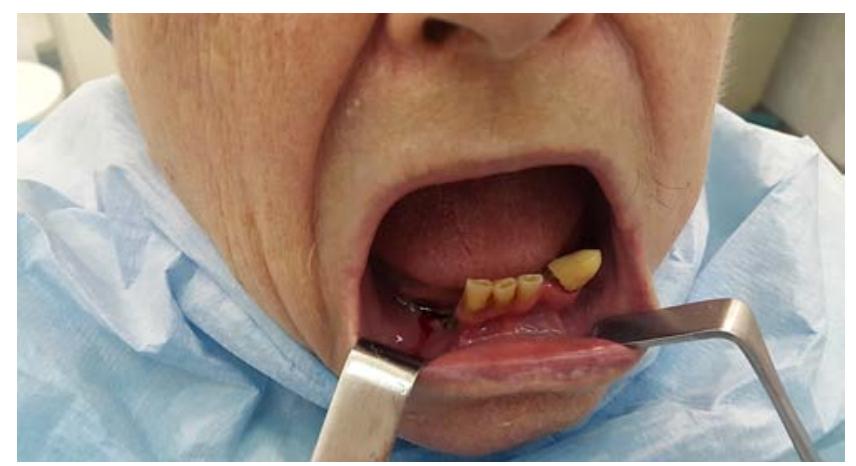

Figure 1. Extraoral image - necrosis of the jaw bone 
the uterus, and for a long time had taken ibandronic acid - a drug from the group of bisphosphonates (BP's). The patient was also burdened with several systemic diseases, including type 1 diabetes, disorders of the cardiovascular system, and neurological disorders.

Radiographs (pantomogram, CBCT, CT) showed an osteolytic lesion in area 31- area of right mental foramen with dimensions $36.25 \times 12.38 \mathrm{~mm}$ (Figs. 2, 3, 4, 5, 6, 7, 8).

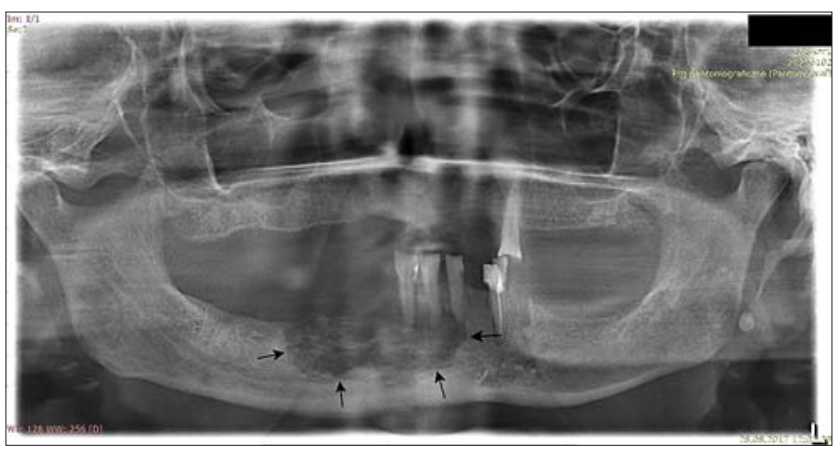

Figure 2. Panoramic radiograph - osteolysis in area 31 - area of right mental foramen; teeth present: $23,33,31,41,42$

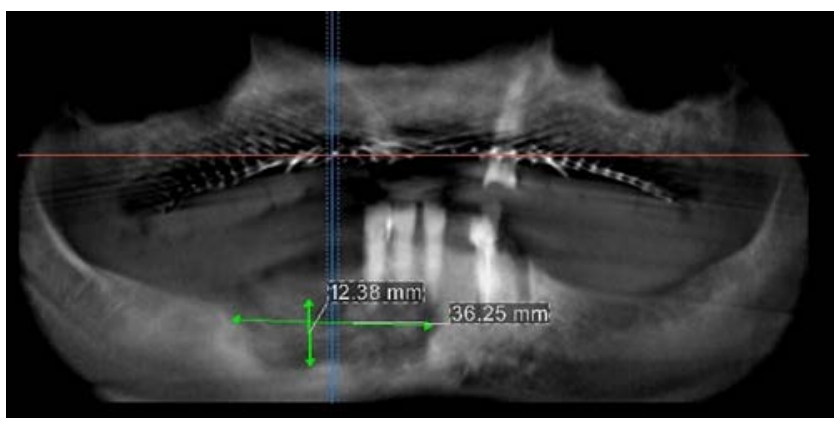

Figure 3. $\mathrm{CBCT}$ - osteolytic lesion, dimensions: $12.38 \mathrm{~mm} \times 26.25 \mathrm{~mm}$

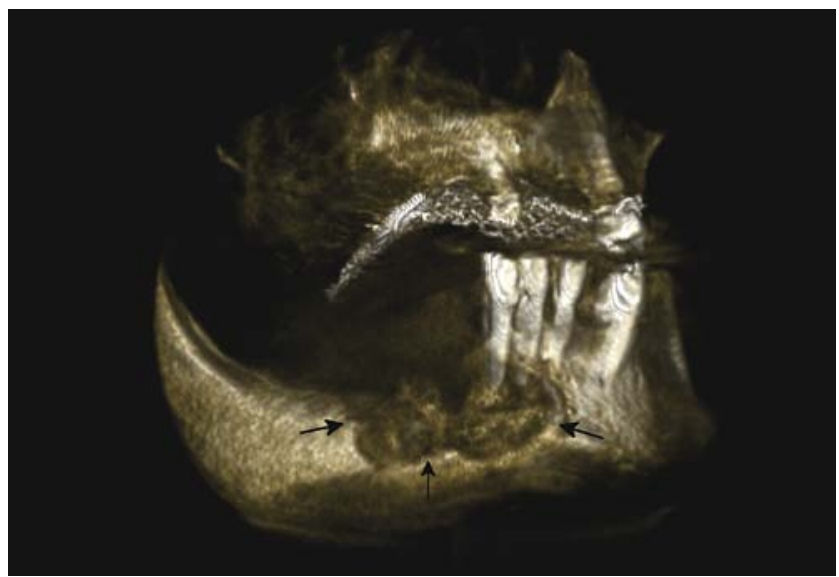

Figure 4. 3D reconstruction of $C B C T$ image - osteonecrotic focus

Under local anaesthesia, 2\% lignocaine with noradrenaline, a superficial revision of the lesion was performed and a specimen taken for histopathological examination. Pathomorphological diagnostics showed actinomycotic bone inflammation. After three months, under general anaesthesia, segmental marginal resection of the mandible was performed. Teeth 33, 31, 41 and 42 affected by the pathological lesion and tooth 33 were removed. (Figs. 9,10). The result of the study confirmed actinomycotic bone inflammation. After the surgery, 3-cycle Ospen therapy was used for a period of eight days. $2 \times 1 \mathrm{mln}$ IU at intervals of one month. During the one month follow-up, check-ups showed healing of the mucosa and no pain. (Fig. 11).

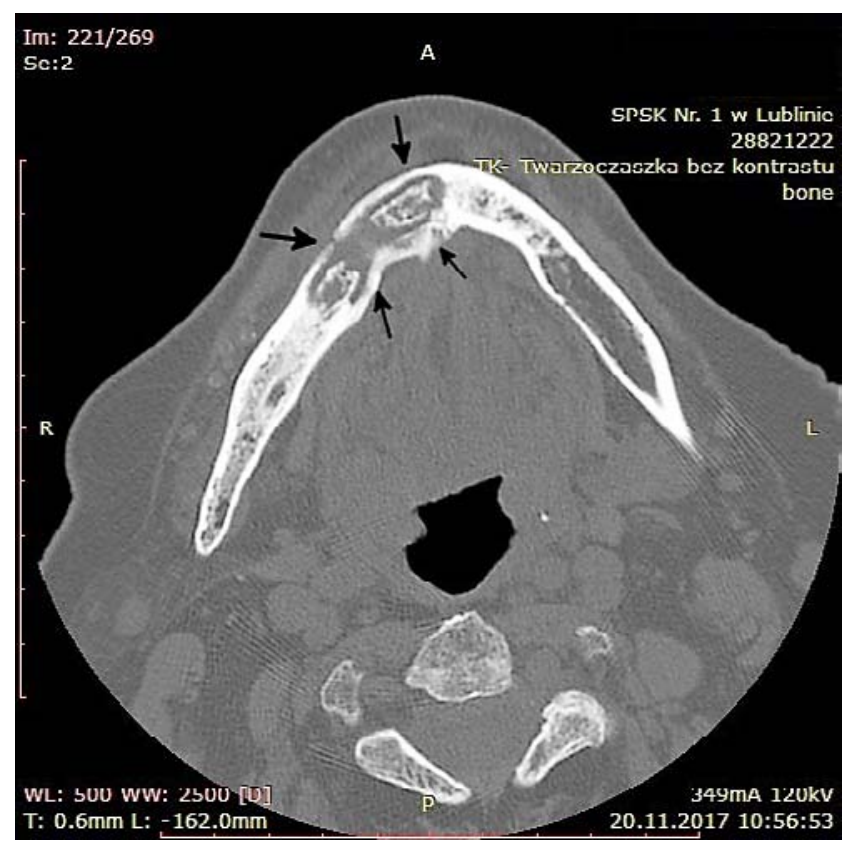

Figure 5. TK - axial section: osteonecrosis

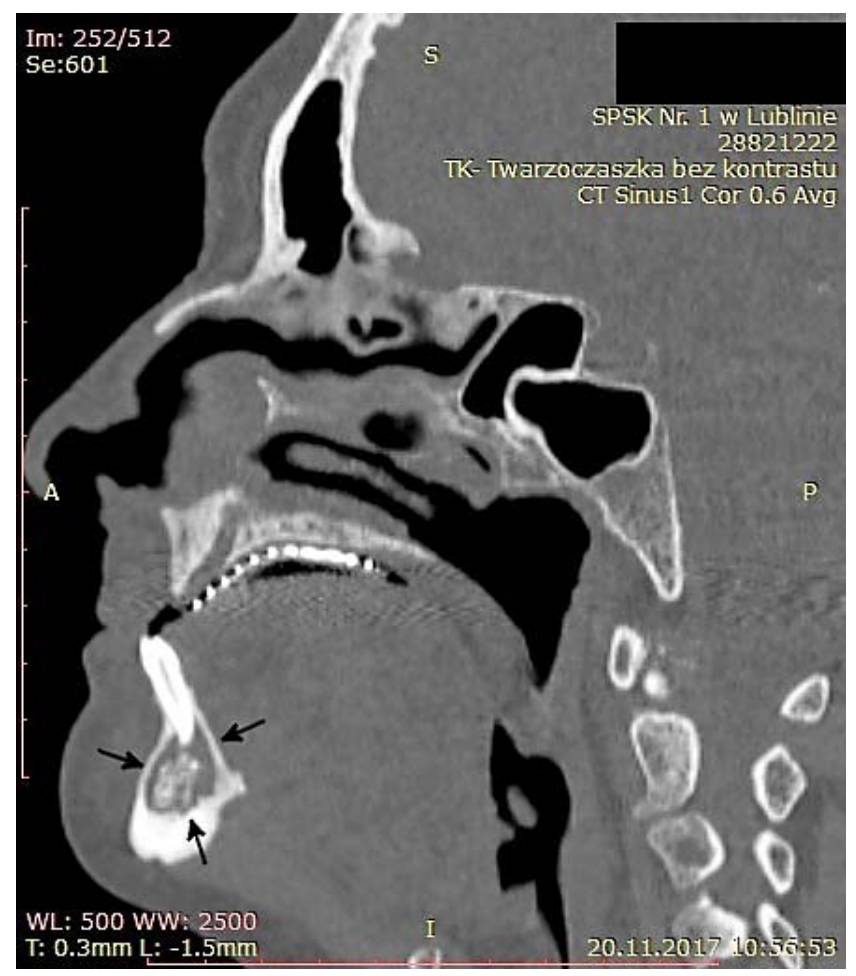

Figure 6. TK - sagittal section: osteonecrosis with bone sequester 


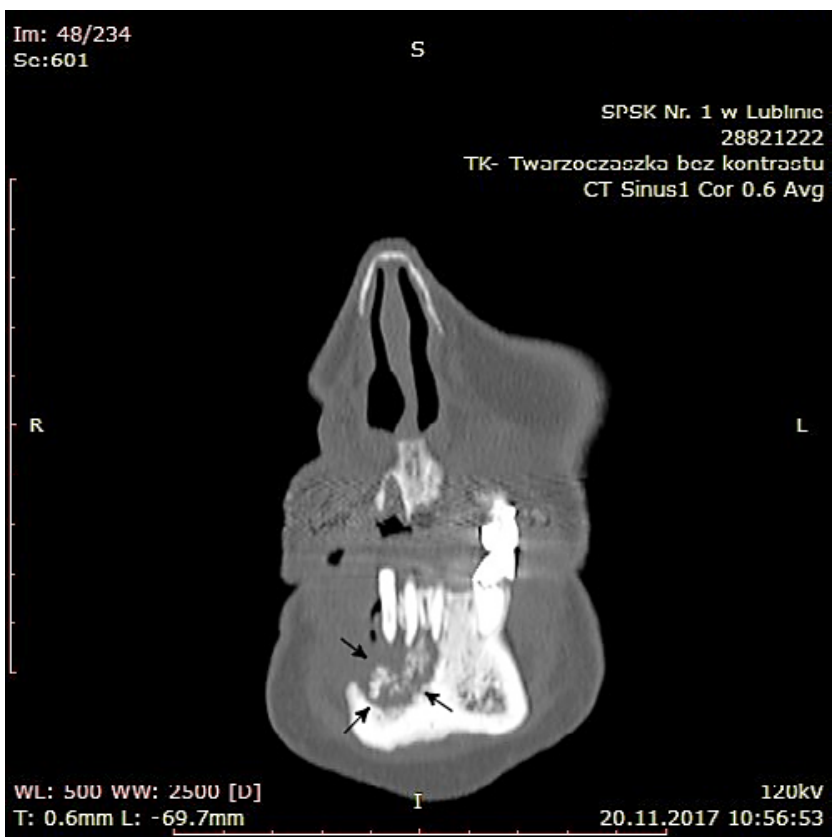

Figure 7. TK - frontal section: osteonecrosis including teeth $31,41,42$

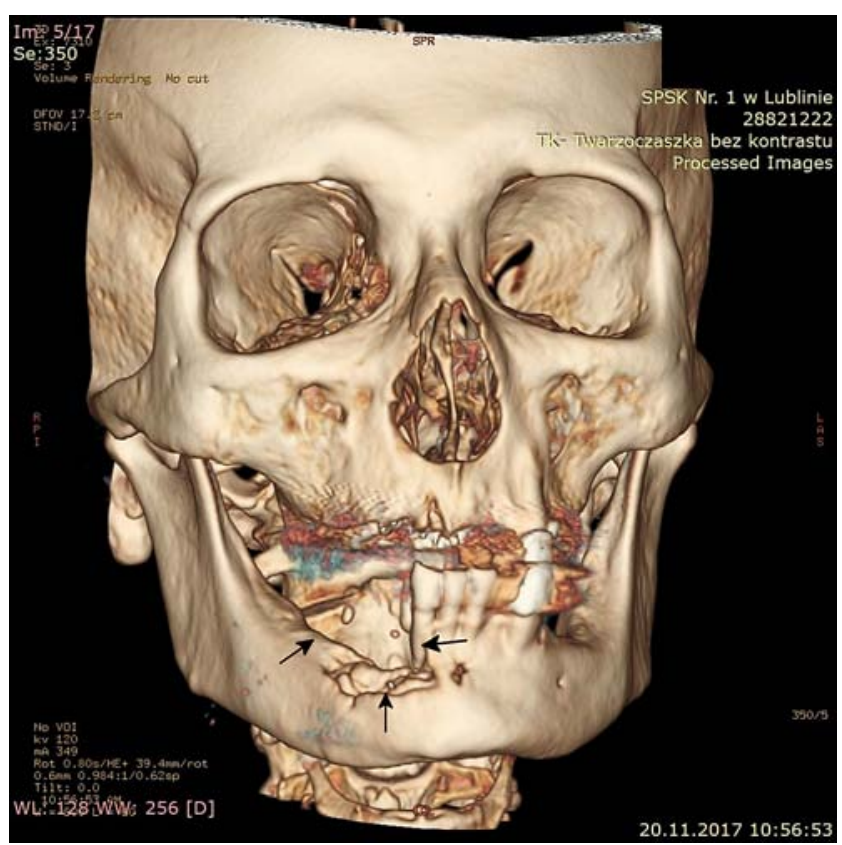

Figure 8. 3D reconstruction of TK image - osteonecrosis

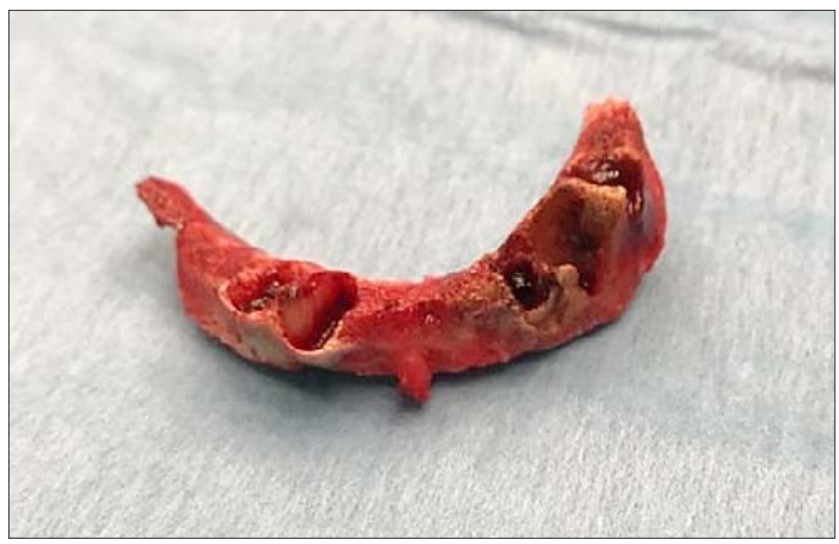

Figure 9. Removed sequestration of mandible

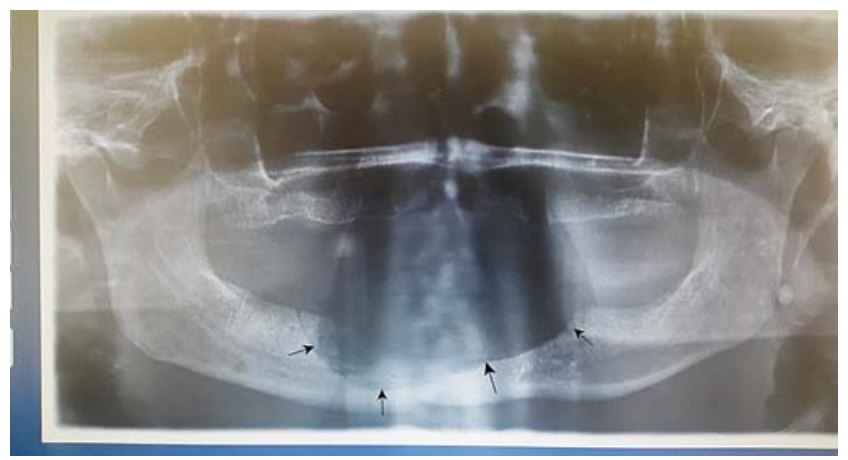

Figure 10. Panoramic radiograph - state after surgical sequestrotomy of mandible

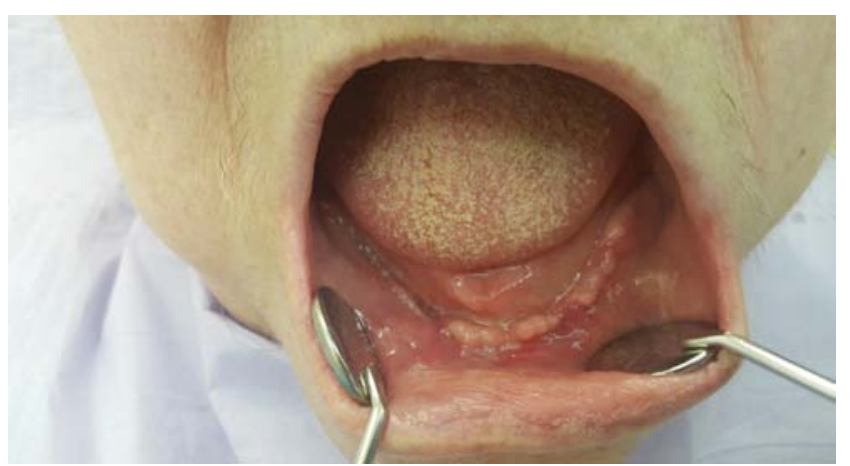

Figure 11. State after healing - follow-up examination one month after surgery

\section{CONCLUSION}

1. In advanced cases, surgical treatment is indicated sequestration of inflammatory focuses supported with antibiotic therapy.

2. Patients receiving biphosphonates should be monitored for development of BRONJ complicated with Actinomycosis.

3. Treatment of Actinomycosis with antibiotics therapy is necessary.

\section{DISCUSSION}

BRONJ is a serious side-effect of treatment with these drugs. Diagnosis of the disease is based on the presence of exposed bone that does not heal after eight weeks, previous or current biphosphonate therapy, and no radiotherapy in the craniofacial region. The concomitant occurrence of actinomyces infection with BRONJ is essential in pathogenesis, and timely actinomycosis-specific treatment may improve outcome [8].

The analysis carried out by Ho-Gul Jeong et al. showed that the occurrence of BRONJ was more than five times more frequent in patients taking bisphosphonates for more than three years ( $>3$ years $7.96 \%,<3$ years $1.43 \%$ ) [5]. Ruggiero et al. and Nocolau-Ioannou et al. came to similar conclusions [6,12]. A longer duration is also associated with their accumulation in bones, where they can survive even 12 years [6]. This explains the possibility of the appearance of BRONJ symptoms after discontinuation of bisphosphonate therapy in the presented case report.

Age is also a factor favouring the occurrence of osteonecrosis of the jaws. Studies by Ho-Gul. Jeong et al. and Hellstein et al. show that people over the age of 65 are more prone to BRONJ 
$[5,18]$. This has also been demonstrated in other studies $[2$, $6,7]$. BRONJ occurs more often in the mandible, which is a consequence of its worse blood supply compared to the maxilla. It is believed that the type of drug administered does not matter; however, Nicolaou-Ioannou indicates an increased risk during therapy with the drugs Zomera and Aredia [12].

Additional factors affecting the decreased resistance of jaw bones include: type 1 diabetes, use of glucocorticoids, autoimmune diseases, disorders involving hematopoiesis, renal dysfunction, peripheral vascular abnormalities, as well as alcohol abuse, smoking and obesity $[4,6,12]$.

In the context of the above-presented case, the correlation of the occurrence of Actinomyces infection with osteonecrosis should be mentioned. The presence of bacteria in necrotic bone has been proven, especially those of the genus Actinomyces. Their appearance may be associated with an increased risk of BRONJ recurrence and an uncertain prognosis after surgical treatment [2]. The surgical procedure itself, which is not combined with appropriate antibacterial therapy, will not be beneficial if BRONJ is complicated by Actinomyces infection. [8].

Preventive treatment of BRONJ is extremely important and lies within the responsibility of both the dentist and the attending physician. Examination of the oral cavity before the beginning of bisphosphonate therapy should be very accurate, and possible sanitisation must be carried out before treatment, in order that the wounds have time to heal [6]. Sanchis believes that when taking BF's, procedures such as simple conservative treatment, endodontic treatment and tooth splinting are allowed [19]. However, when tooth extraction is unavoidable, special procedures must be implemented to prevent the development of BRONJ [13]. These are based on discontinuation of the drug administration before and after the procedure (drug holiday). It is recommended for patients receiving oral bisphosphonates, for more than four years, and for those who have additional risk factors, such as glucocorticoid therapy, diabetes, etc.

\section{REFERENCES}

1.Gómez Font R, Martínez García ML, Olmos Martínez JM, Osteochemonecrosis of the Jaws due to Bisphosphonate Treatments. Update, Med Oral Patol Oral Cir Bucal. 2008 May 1; 13(5): E318-24.

2. Kim T-H, Seo W-G, Koo C-H, Lee J-H. Evaluation of the predisposing factors and involved outcome of surgical treatment in bisphosphonaterelated osteonecrosis of the jaw cases including bone biopsies. Journal of the Korean Association of Oral and Maxillofacial Surgeons. 2016; 42(4): 193-204. doi:10.5125/jkaoms.2016.42.4.193

3. Campisi G, Fedele S, Fusco V, et al. Epidemiology, clinical manifestations, risk reduction and treatment strategies of jaw osteonecrosis in cancer patients exposed to antiresorptive agents, Future Oncol. 2014 Feb; 10(2): 257-75. doi: $10.2217 /$ fon.13.211

4. Gawęda A, Czerkies R, Trzaskowska E, Tomaszewski T. Bisphosphonaterelated osteonecrosis of the jaw in a 66-year-old female - Case report. mJ Pre-Clin Clin Res. 2017; 11(2): 162-166. doi: 10.26444/jpccr/80726

5. Jeong H-G, Hwang JJ, Lee J-H, Kim YH, Na JY, Han S-S. Risk factors of osteonecrosis of the jaw after tooth extraction in osteoporotic patients on oral bisphosphonates. Imaging Science in Dentistry. 2017; 47(1): 45-50. doi:10.5624/isd.2017.47.1.45

6. Ruggiero SL, Dodson TB, Fantasia J, et al. American Association of Oral and Maxillofacial Surgeons position paper on medication-related osteonecrosis of the jaw-2014 update. J Oral Maxillofac Surg. 2015; 73: 1440. doi: 10.1016/j.joms.2015.04.023.

7. Nair V, BRONJ: What Should a Dental Surgeon Know?, International Medical Journal Vol. 24, No. 1, pp. 117-119, February 2017.

8. Naik NH, Russo TA, Bisphosphonate-Related Osteonecrosis of the Jaw: The Role of Actinomyces, Clin Infect Dis. 2009 Dec 1; 49(11): 1729-32. doi: $10.1086 / 648075$

9. Fukushima Y, Enoki Y, Nakaoka C, et al. Usability of surgical treatment in cases of bisphosphonate-related osteonecrosis of the jaw stage 2 with sequestrum. Annals of Maxillofacial Surgery. 2015; 5(1): 67-70. doi: 10.4103/2231-0746.161067

10. Vidal-Gutiérrez X, Gómez-Clavel JF, Gaitán-Cepeda LA. Dental extraction following zoledronate, induces osteonecrosis in rat's jaw. Med Oral Patol Oral Cir Bucal. (2017), doi: 10.4317/medoral. 21609

11. Bagan L, Jiménez Y, Leopoldo M, Rubert A, Bagan J. Exposed necrotic bone in 183 patients with bisphosphonate-related osteonecrosis of the jaw: Associated clinical characteristics. Med Oral Patol Oral Cir Bucal. (2017), doi: 10.4317/medoral.22133

12. Nicolaou-Ioannou Z, Kaplan I, Nonresolving Osteomyelitis of the Maxilla and Maxillary Sinus after Long-term Use of Oral Bisphosphonates, International Journal of Head and Neck Surgery, May-August 2010; 1(2): 107-110.

13. Omami M, Ben Ali R, Sioud S, Hentati H, Selmi J, Incidence and Risk Factors of Bisphosphonate-Related Osteonecrosis of the Jaw Following Tooth Extraction: A Systematic Review, Sch J App Med Sci. 2017; 5(10F): 4276-4283. doi 10.21276/sjams.2017.5.10.90

14.López-D’alessandro E, Mardenlli F, Paz M. Oral bisphosphonateassociated osteonecrosis of maxillary bone: A review of 18 cases. Journal of Clinical and Experimental Dentistry. 2014; 6(5): e530-e534. doi: $10.4317 /$ jced. 51694

15. Black DM, Reid IR, Boonen S, et al. The Effect of 3 Versus 6 Years of Zoledronic Acid Treatment of Osteoporosis: A Randomized Extension to the HORIZON-Pivotal Fracture Trial (PFT). Journal of Bone and Mineral Research. 2012; 27(2): 243-254. doi:10.1002/jbmr.1494

16. Leite AF, Ogata Fdos S, de Melo NS, Figueiredo PT, Imaging Findings of Bisphosphonate-Related Osteonecrosis of the Jaws: A Critical Review of the Quantitative Studies, Int J Dent. 2014; 2014: 784348 doi: 10.1155/2014/784348. Epub 2014 Jun 11.

17. Krstevska S, Stavric SG, Cevrevska L, et al. Osteonecrosis of the Jaw After Bisphosphonates Treatment in Patients with Multiple Myeloma. Medical Archives. 2015; 69(6): 367-370. doi: 10.5455/medarh.2015.69.367-370.

18. Hellstein JW, Adler RA, et al. Managing the care of patients receiving antiresorptive therapy for prevention and treatment of osteoporosis Executive summary of recommendations from the American Dental Association Council on Scientific Affairs, JADA 2011; 142(11): 612431251.

19. Sanchis JM, Bagán JV, Murillo J, Díaz JM, Asensio L, Risk of developing BRONJ among patients exposed to intravenous bisphosphonates following tooth extraction. Quintessence Int. 2014 Oct; 45(9): 769-77. doi: 10.3290/j.qi.a32243. 